\title{
Innovative Energy Elevator: a Physics and Engineering Wonder!
}

\section{Prof. Bala Maheswaran, Northeastern University}

Bala Maheswaran, PhD Northeastern University 367 Snell Engineering Center Boston, MA 02115

Mr. Cristian Scott Stransky, Northeastern University

Computer science and computer engineering student enrolled in Northeastern, graduating 2020.

Dr. Haridas Kumarakuru, Northeastern University

Department of Physics, College of Science, 100 Forsyth street, Boston, MA 02115 E.Mail: h.kumarakuru@neu.edu 


\title{
Innovative Energy Elevator: A Physics and Engineering Wonder!
}

\author{
Cristian Stransky ${ }^{1}$, Damien Dumayas ${ }^{1}$, Tianyi Dai ${ }^{1}$, Paponvich Chattrapronvichian ${ }^{1}$, \\ Haridas Kumarakuru ${ }^{2}$ and Bala Maheswaran ${ }^{1}$ \\ College of Engineering ${ }^{1}$ \\ Department of Physics ${ }^{2}$ \\ Northeastern University
}

\begin{abstract}
In the past decades, people are using more electricity in their daily life; therefore, it becomes crucial to research for methods to increase energy production, especially from day to day activities. Considering our average daily routine and using simple physics concepts, we have designed a method to harness energy from the movement of an elevator. In this paper, we describe a miniature model of an elevator and its shaft through 3D printing. We wrapped a copper wire around the structure and placed a stack of magnets in the elevator. On its downward movement, our project successfully generated current through the copper wire by electromagnetic induction and basic physics. We recorded the electric potential through our Sparkfun circuit and then used Matlab to analyze our results. Our primary goal was to prove that Faraday's theory could be applied to an elevator to produce electric potential, and our secondary goal was to optimize the electric potential generated by the process for the practical use. We hope that this model could either work in the real world or lay the groundwork for the next generation.
\end{abstract}

\section{Introduction}

Since the end of 19th century, there have been many scientific discoveries and inventions that were groundbreaking for humanity, such as the invention of the steam engine, automobile, the construction of the skyscraper, and the aircraft. Nevertheless, these significant developments also result in the tremendous depletion of the world's resources. In the US, the use of petroleum has risen from less than one quadrillion $\left(10^{15}\right)$ BTU in 1900, to 41 quadrillion BTU in 2000, and the trend is likely to increase significantly in this century ${ }^{1}$. Therefore, the development of renewable energy and the research for new sources of energy has become more necessary ${ }^{2-7}$.

One of the objectives of the Experiential Engineering Education and this paper is to reform engineering education by moving away from the boundaries of traditional classroom-based approaches to project- concept- and team-based, and skill- and knowledge-integrated approaches using real world situations. This new teaching approach can improve the effectiveness of engineering education. Introducing new teaching approaches is always a challenging task and has been explored using various tactics, and the detailed work is published in the peer reviewed journals and proceedings ${ }^{8-13}$. For experiential energy innovation team project, we selected magnetism and its application to generate electricity via an innovative approach. Magnetic 
induction was discovered by Michael Faraday in the mid-19th century ${ }^{14-15}$, that is, "any change in the magnetic environment of a coil of wire will cause a voltage in the coil. No matter how the change is produced, the voltage will be generated". The most common technology using magnetic induction is a generator, which is the source providing electricity to most households. We propose to that this concept could be applied to our daily routine to generate electricity.

The Elevator is highly used for vertical transportation. Originally, an elevator was used as a method for serving food from the kitchen to the dining rooms of English Lords' households. The advent of the skyscraper led to the need for development and its popularity at the same time. Therefore, an elevator was installed in almost every building taller than 4 floors. According to National Elevator Inc. ${ }^{16}$, there are more than 900,000 units of elevators in the United States. Based on these facts, we conclude that the elevator is most suitable to test our concept, and we decided to innovate the existing elevator technology to produce energy while it's moving. The elevator's vertical motion is an ideal setup to build a simple educational prototype to produce energy. Regenerating energy from building lift concepts has already been explored and published in an International Journal ${ }^{17}$.

Our approach is to make a smaller model resembling a real elevator. We constructed the model mostly by using 3D printed parts. The design was divided in two main categories: the elevator and the structure. To design the prototype, we considered its strength, aesthetics, cost, and its ability to support large amounts of copper coils around it.

Our main goal is to prove that this model of elevator can generate electricity, and to see if it is profitable compared to the cost. Since this project was an experiential engineering educational project to prove the concept, we did not plan to do further experimentation once the concept is proved, but, we hope that this educational model could lay the groundwork for real world technology development.

\section{Methods and Approach}

To build the model, we considered using LEGO bricks, wood, or 3D printed elements. Our first concern was practicality, that is, whether the model constructed with a particular material would be suitable to test the concept. Second concern was production time and difficulty, and the third concern was aesthetics. Although LEGO ranked high in all three, its cost and low durability made it unsuitable for the project. On the other hand, wood would make a very durable model, but precision and aesthetics were the concerns. We then decided to use Solidworks to design elements with precision and to use a 3D printer to print the parts. We used wood for the base and linen thread as cable.

Our model was divided into two main parts: the elevator and the structure ${ }^{16}$, and the latter was also divided into three parts namely; the floor, the middle, and the top. The change in magnetic 
flux determined the voltage output and depends on the number of coils around the system. Thus, our design had to take into account of methods to wrap the wire around the structure as many times as possible to increase number of turns per unit length.

We designed all the structure pieces with holes at their corners to thread acrylic rods through those holes, and a wooden base to increase the stability. We designed a hollow structure to reduce the printing time and to visualize the movement of the elevator. The elevator was designed to hold magnets inside. The elevator was built to be slightly smaller than the structure outside so that it could move smoothly. We used 28 gauge copper wire and $0.5^{\prime \prime} \times 0.5^{\prime \prime} \times$ $0.125^{\prime \prime}$ of N45 Neodymium magnets (1.3 Teals each). We initially intended to use a DC motor in Sparkfun kit to control the elevator movement. However, the motor turned out to be too weak to lift the elevator with the magnets. Therefore, we manually moved the elevator. The output voltage was connected to Sparkfun circuit to display the amount of voltage on a LCD screen. We used the Matlab program to graph the voltage.

\section{Design Details}

The elements for the prototype of the elevator, and the structure were design using Solidworks. The dimension of the floor part shown in Figure 1a, is $3 \mathrm{~cm} \times 3 \mathrm{~cm} \times 3 \mathrm{~cm}$, with a base thickness of $0.5 \mathrm{~cm}$. The area of the holes in the main square is $2 \mathrm{~cm} \times 2 \mathrm{~cm}$. The holes in each corner had a radius of 0.125 inch which is equal to the radius of the acrylic rod used to keep the structure stable.

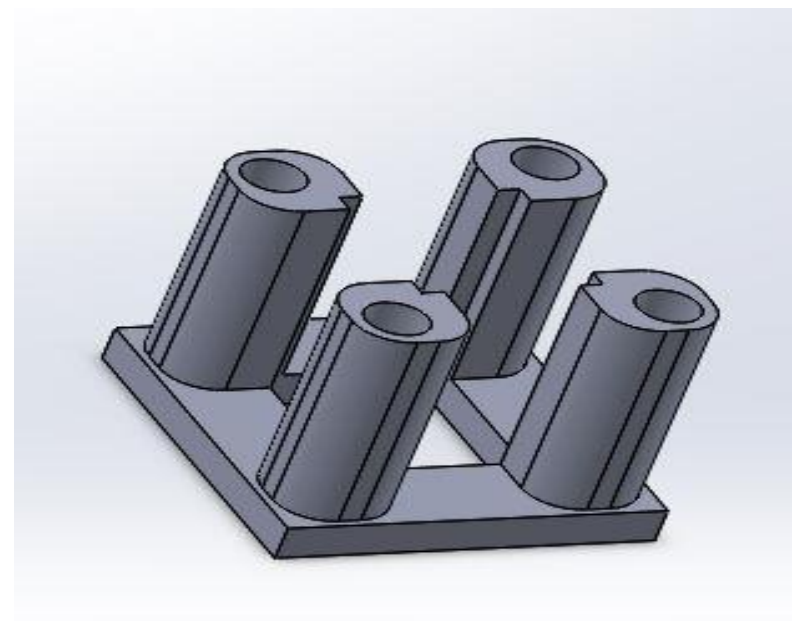

Figure 1a: The floor part of the model

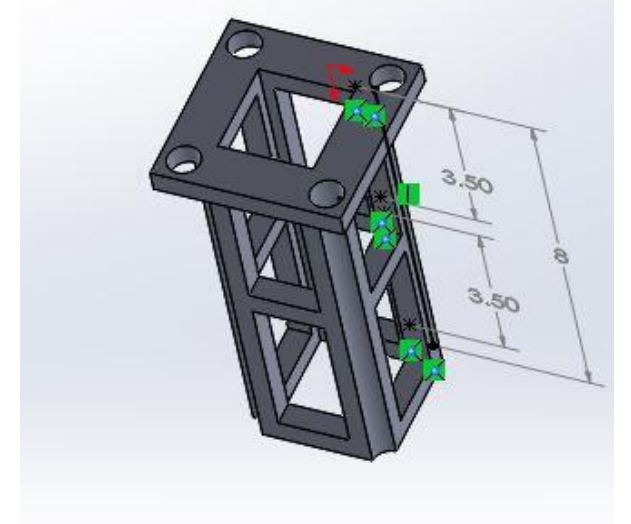

Figure 1b: The middle part of model

The middle portion shown in Figure $1 \mathrm{~b}$ has dimensions of $2.5 \mathrm{~cm} \times 2.5 \mathrm{~cm} \times 8 \mathrm{~cm}$. The square hole has an area of $2 \mathrm{~cm} \times 2 \mathrm{~cm}$. The corner extrudes up and fillet with the radius of $0.125 \mathrm{inch}$.

The top portion of the structure in Figure 1c, is similar to the floor part; however, instead of a big square hole, it has a hole in the shape of the axle. We 3D printed the axle along with this part. 
The elevator in shown in Figure 1d, has a dimension of $1.3 \mathrm{~cm} \times 1.8 \mathrm{~cm} \times 3 \mathrm{~cm}$ and is hollow inside. It had four hooks on the four corners at the top to attach the string.

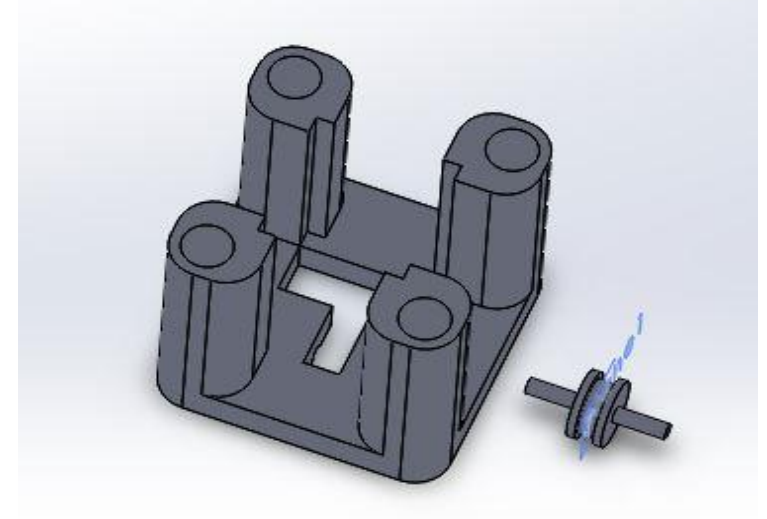

Figure 1c: The top part of model

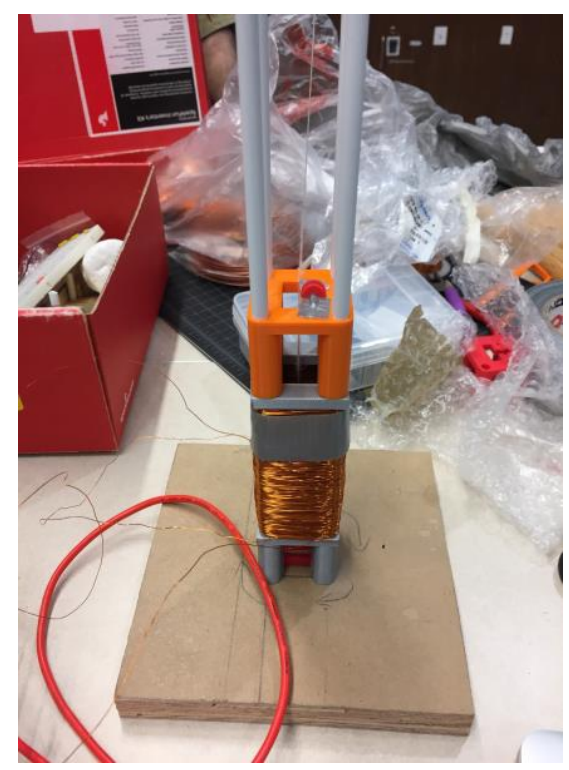

Figure 1e: The Model

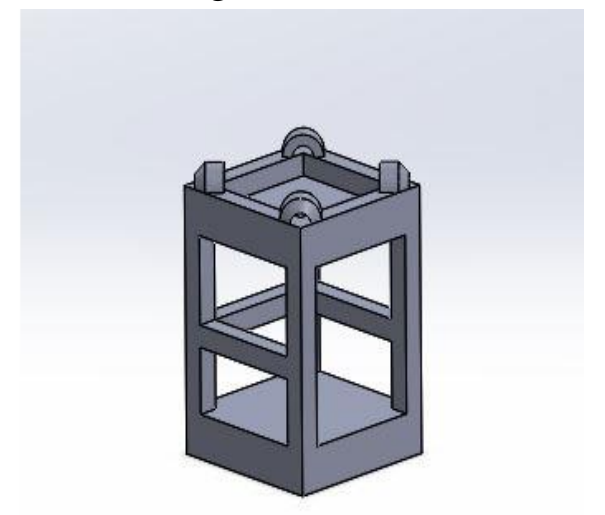

Figure 1d: Elevator

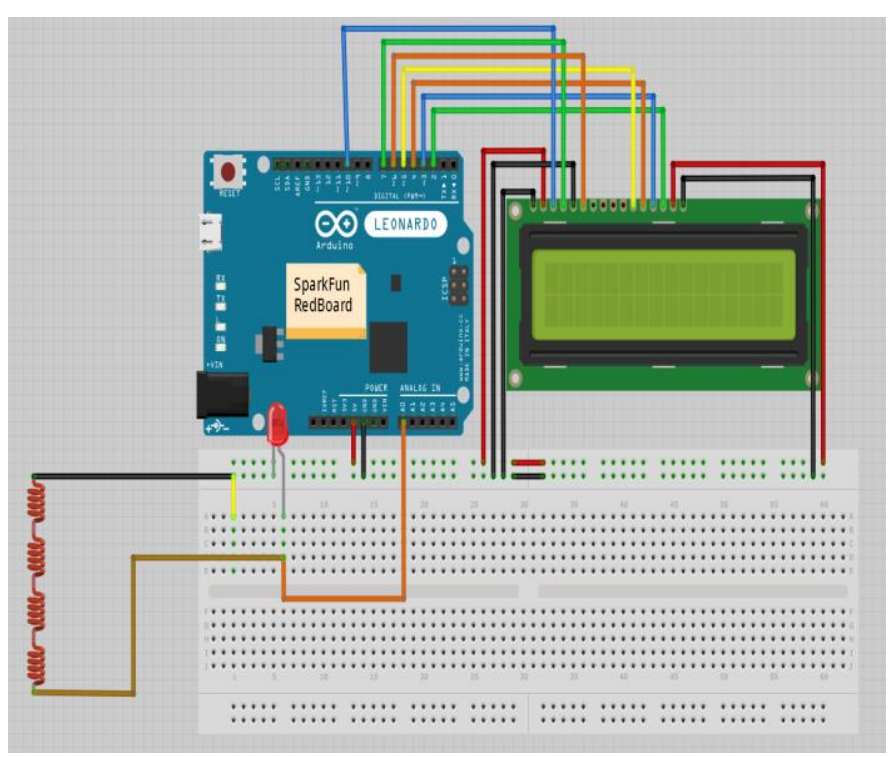

Figure 2: Sparkfun Circuit

The assembled prototype is shown in Figure 1e. It has 1000 turns of 28 gauge copper wire in the middle. A linen thread is attached to the elevator, and we placed 6 Neodymium (N45) magnets inside the elevator.

To test the system, we released the elevator through the square holes between the middle part and top part. Since the elevator was slightly smaller than the holes, it was able to move smoothly through the hole. The model was strong, well-made, and did not tumble when the elevator moved at various speeds. The Sparkfun circuit in Figure 2 was used as voltmeter ${ }^{18-19}$ to collect data, the 
data was displayed on the computer screen using the Matlab program and on the LCD screen as shown in Figure 3b.

\section{Results}

While waiting for the 3D printer to print the middle portion, we constructed the initial prototype without the middle part. This prototype shown in Figure 3a had 20 gauge copper wire wrapped around 64 times. We used multimeter to collect the data. At high speed, that is a flight time of $0.5 \mathrm{~s}$, the system generated $2 \mathrm{mV}$ of electricity. This test proved that the Faraday's law could be applied to the elevator system to generate electricity, but the output voltage is not large enough.

After printing all the elements with a 3D printer, we construct the final prototype. In this case, we wrapped the 28 gauge copper wire a 1000 times. First, we tested the system by moving the elevator at the same speed as we did earlier and were able to optimize the electric potential at 0.6 - 2 V. At 0.2 second flight time, we were able to light the LED momentarily as shown in Figure $3 b$ (red LED).

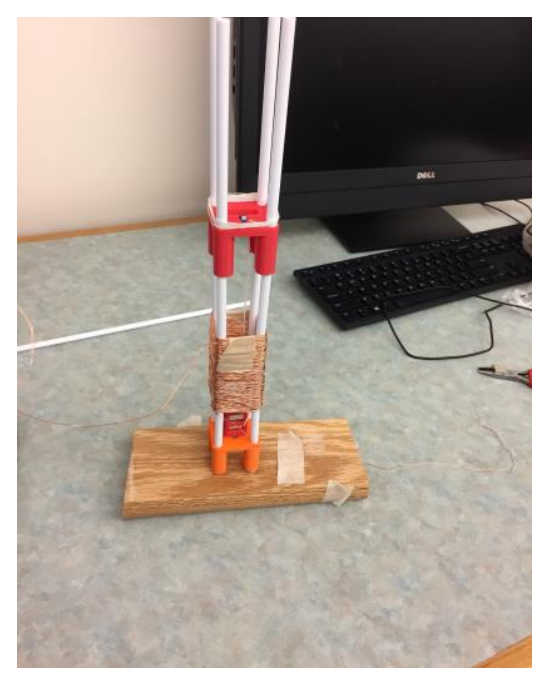

Figgure 3a: The Prototype

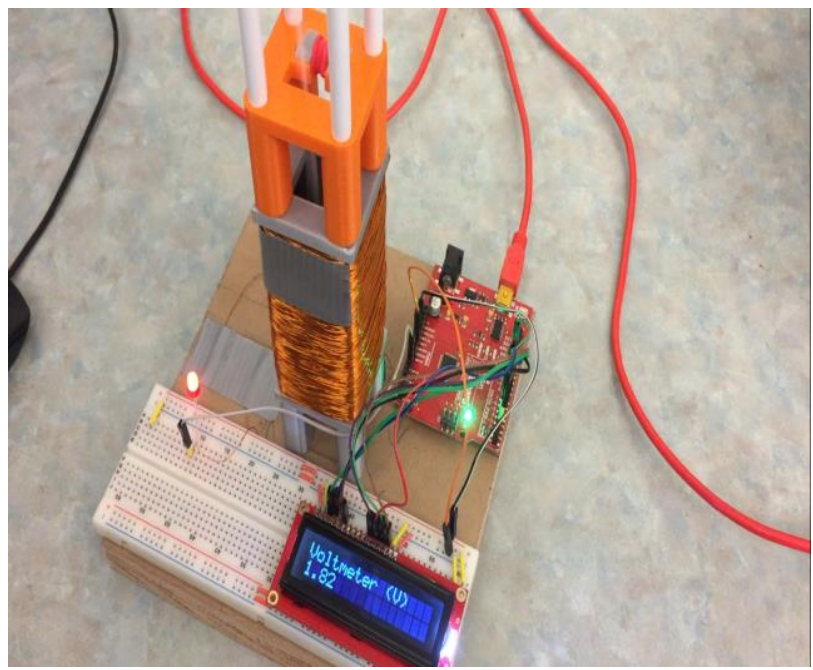

Figure 3b: LED lighted up and LCD display the voltage

Next, we moved the elevator at different speeds namely: slow configuration (Figure 4a - flight time is 2 seconds), medium configuration (Figure $4 \mathrm{~b}$ - flight time is 1 second), fast configuration (Figure $4 \mathrm{c}$ - flight time is 0.5 second). In each configuration, electric voltage was generated by the elevator. As the speed increases the voltage generated by the elevator also increases. 

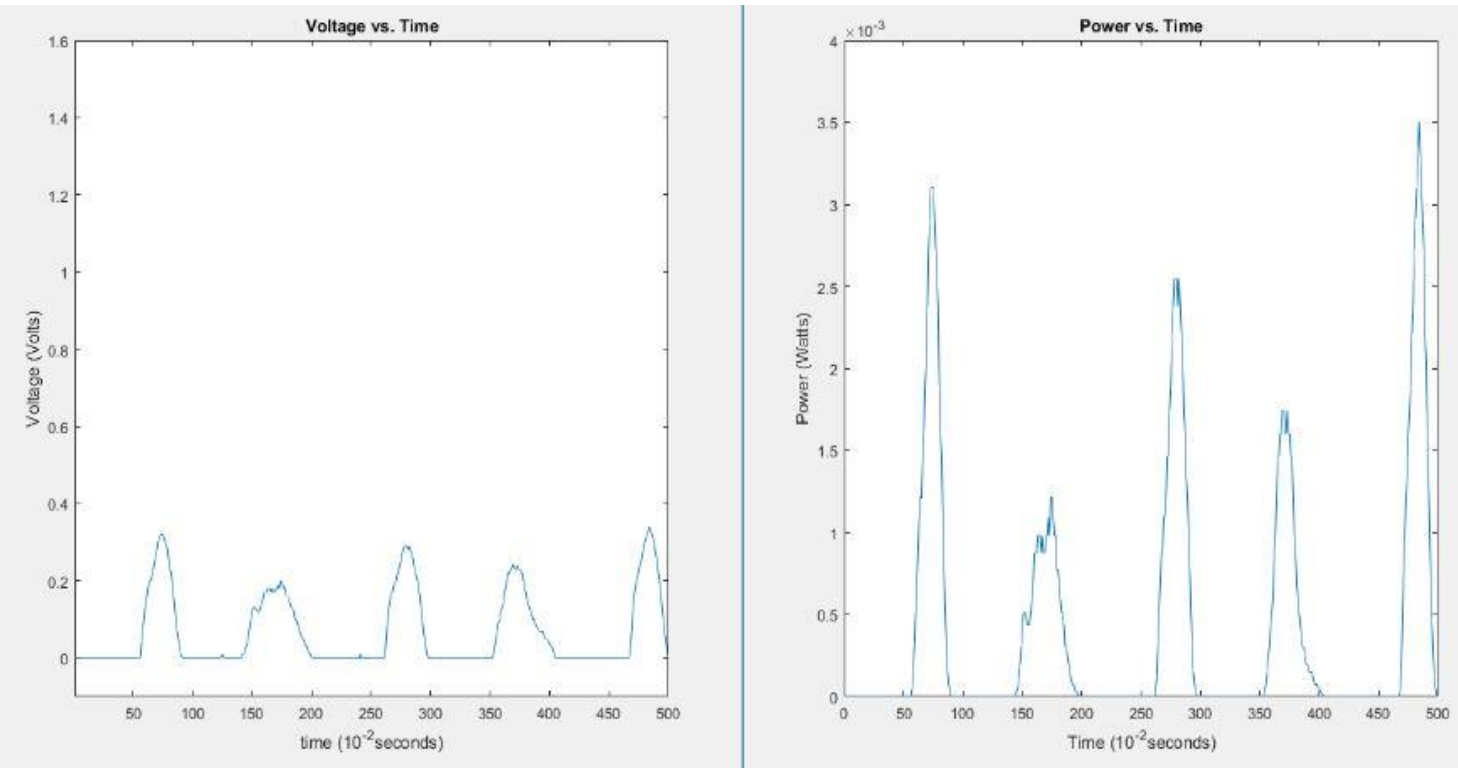

Figure 4a: Slow Speed Configuration

The maximum electrical potential generated in the slow speed configuration was $0.34 \mathrm{~V}$ and the maximum power was $3.5 \mathrm{~mW}$.
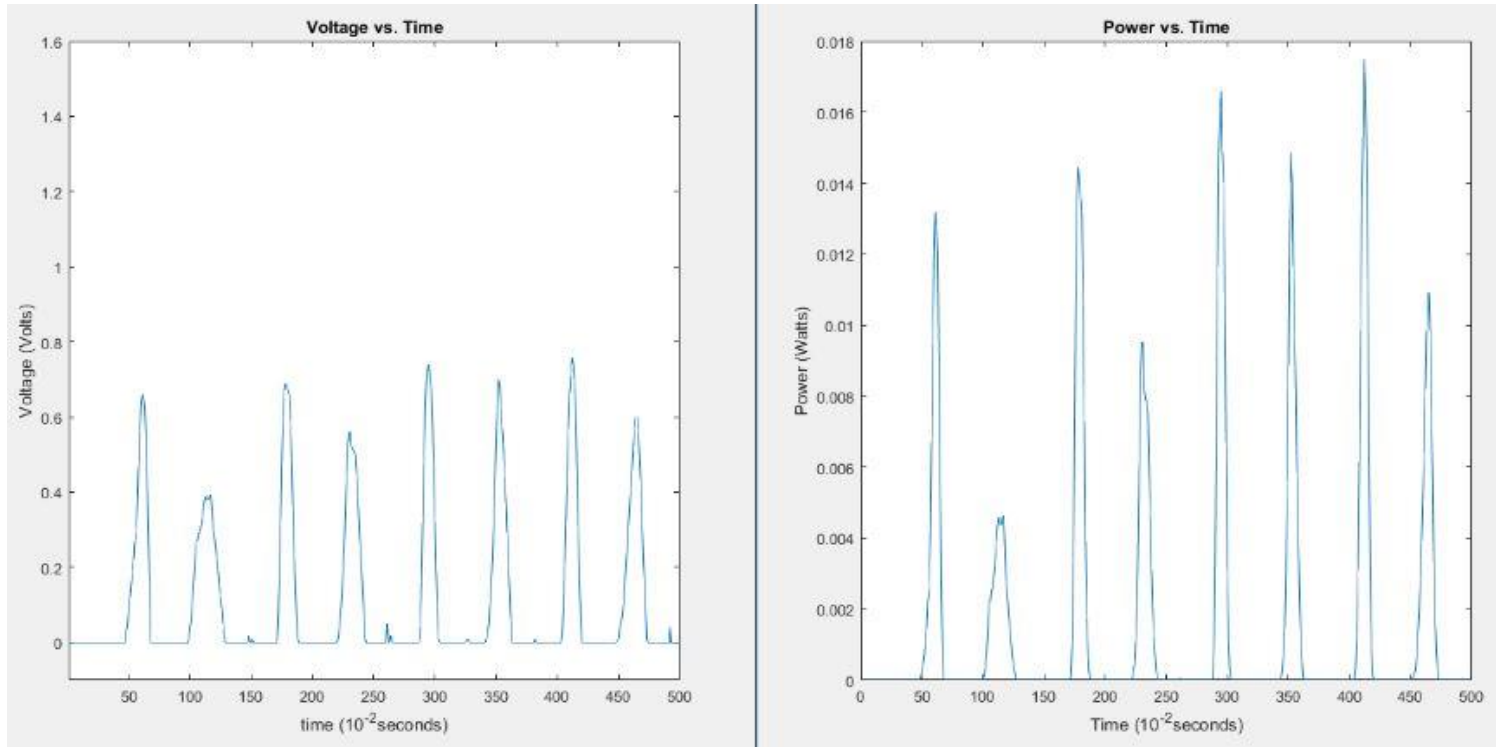

Figure 4b: Medium Speed Configuration

The maximum electrical potential generated in the medium speed configuration was $0.76 \mathrm{~V}$ and the maximum power generated was $17.5 \mathrm{~mW}$. 

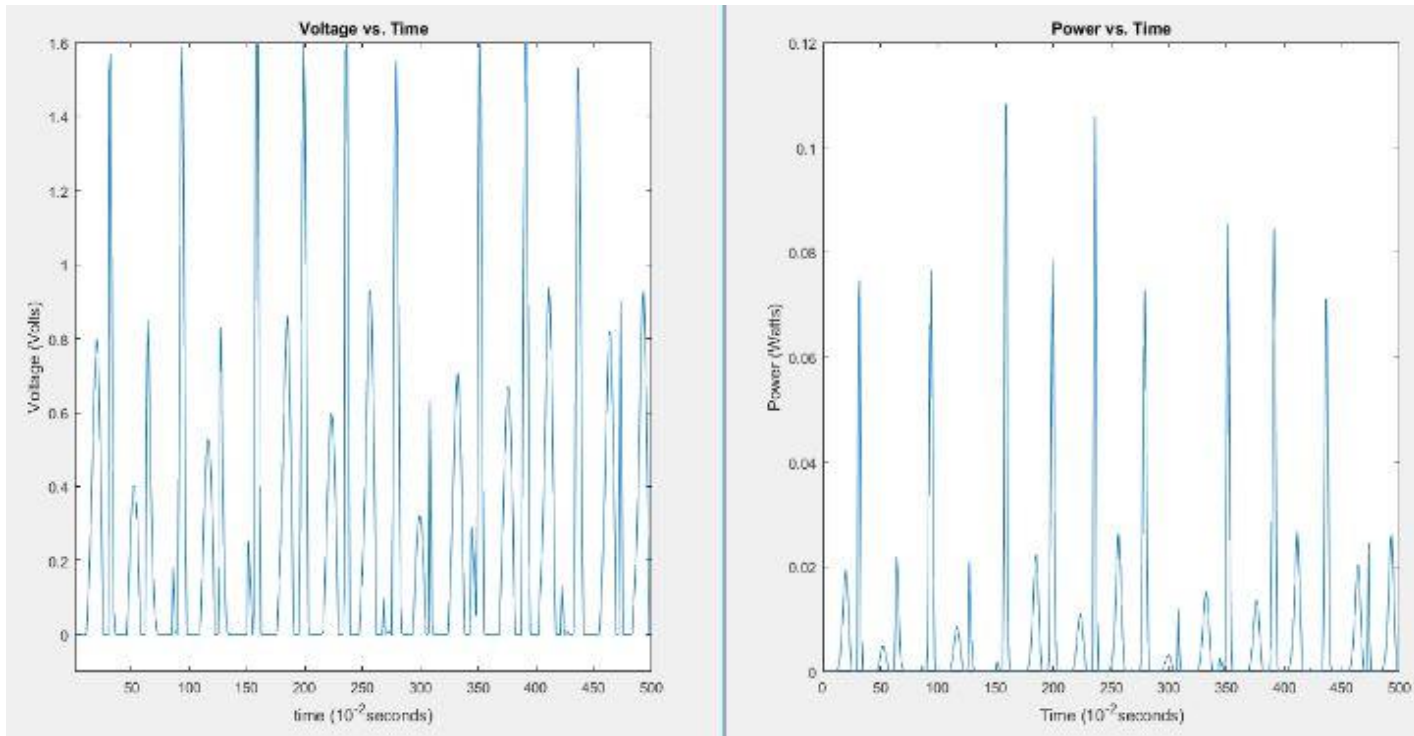

Figure 4c: Fast Speed Configuration

The maximum electrical potential generated in the fast speed configuration was $1.89 \mathrm{~V}$ and the maximum power was $108.2 \mathrm{~mW}$.

Finally, we placed different numbers of magnets in the elevator (2, 6, 10 magnets - results shown in figures $5 \mathrm{a}, 5 \mathrm{~b}, 5 \mathrm{c}$ respectively) and moved it through the coil at the medium speed configuration. We found that as the number of magnets in the elevator increased, more electric voltage was produced.
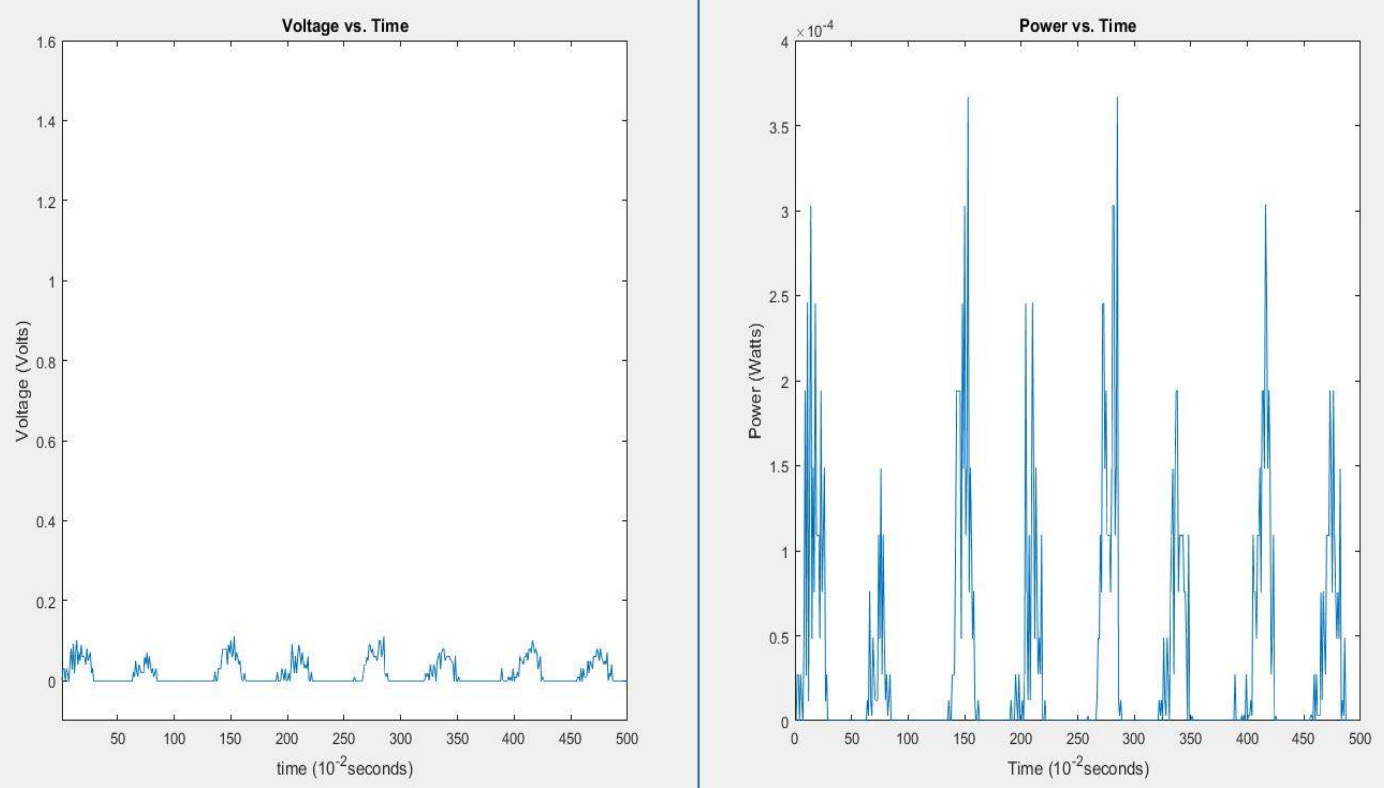

Figure 5a: 2 Magnets

The maximum electrical potential generated in this medium speed configuration was $0.11 \mathrm{~V}$ and the maximum power was $0.34 \mathrm{~mW}$. 

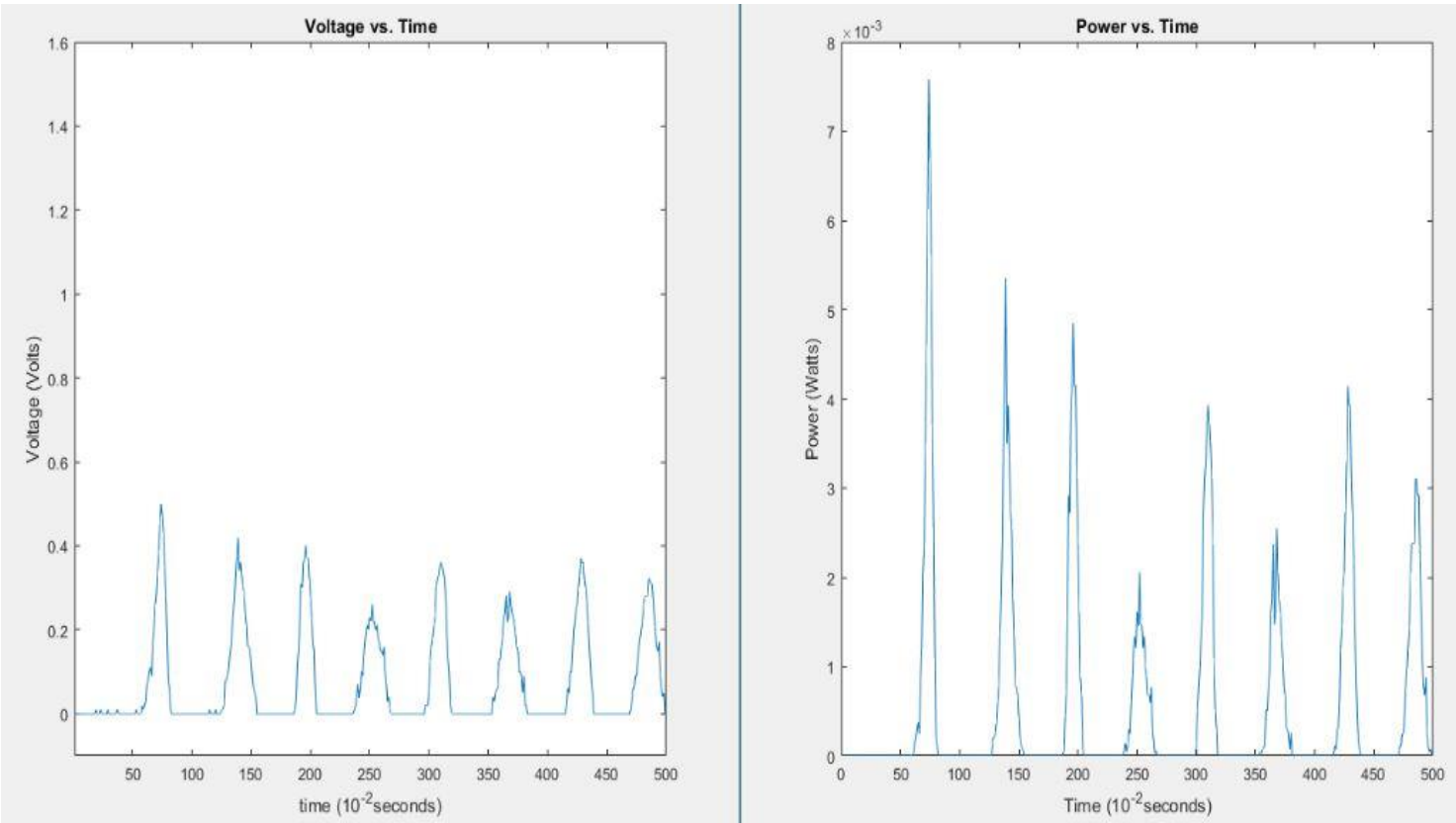

Figure 5b: 6 Magnets

The maximum electrical potential generated in this medium speed configuration was $0.5 \mathrm{~V}$ and the maximum power was $7.6 \mathrm{~mW}$.
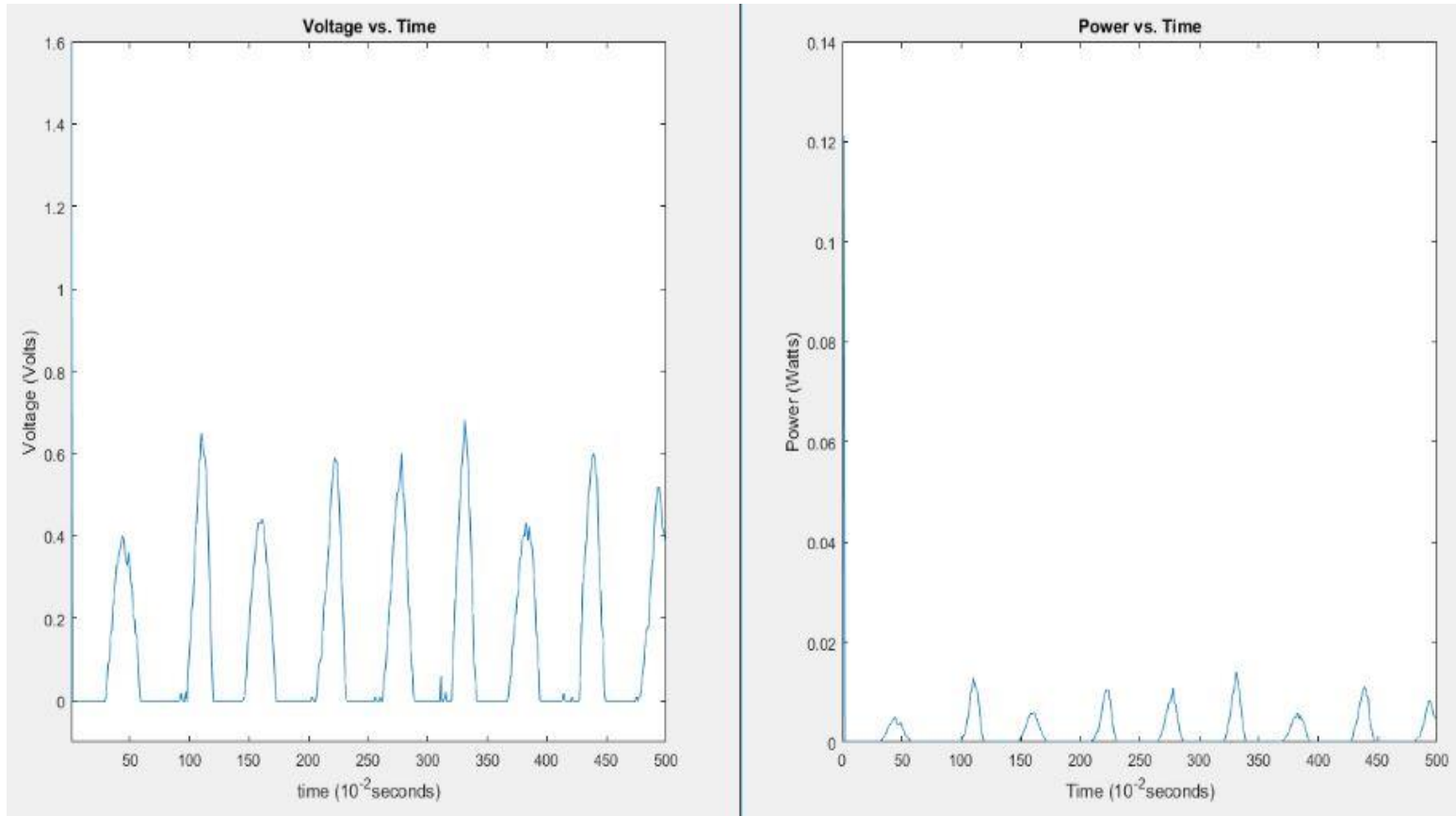

Figure 5c: 10 Magnets

The maximum electrical potential generated in this configuration was $2 \mathrm{~V}$ and the maximum power was $121.2 \mathrm{~mW}$. 


\section{Discussion}

Overall the prototype worked with satisfying results. We were able to produce enough voltage to light the LED. By increasing the speed of the elevator or number of coils around the structure, we could generate substantial voltage with the medium speed configuration. The proposed system needs additional work to see whether this would be commercially beneficial for real world application.

The purpose of this experiential learning project via innovation approach is to prove that the innovative concept proposed works. We still need to test this concept in a larger scale model or in a regular elevator to test its practicality. We anticipate that the cost to apply this concept to a real elevator would be high. Cheaper ways to implement it should be explored. Another concern we have is the number of magnets in the elevator. This could be hazardous if passengers carry materials made from metal. We have researched for magnetic shielding by sheeting the floor and wall with nickel; however, that would add more to the cost to the system.

\section{Conclusion}

We conclude that Faraday's Law could be applied to the elevator system to generate electricity; this is a new novel approach to produce energy from a moving elevator. Table1 summarizes the results:

\begin{tabular}{|c|c|c|c|c|c|c|}
\hline Number of & Slow Spee & onfiguration & Medium Sp & nfiguration & Fast Speed & figuration \\
\hline & $\begin{array}{c}\text { Maximum } \\
\text { Electrical } \\
\text { Potential } \\
\text { Generated } \\
(\mathrm{mV})\end{array}$ & $\begin{array}{c}\text { Maximum } \\
\text { Power } \\
\text { Generated } \\
(\mathrm{mW})\end{array}$ & $\begin{array}{c}\text { Maximum } \\
\text { Electrical } \\
\text { Potential } \\
\text { Generated } \\
(\mathrm{mV})\end{array}$ & $\begin{array}{c}\text { Maximum } \\
\text { Power } \\
\text { Generated } \\
(\mathrm{mW})\end{array}$ & $\begin{array}{c}\text { Maximum } \\
\text { Electrical } \\
\text { Potential } \\
\text { Generated } \\
(\mathrm{mV})\end{array}$ & $\begin{array}{l}\text { Maximum } \\
\text { Power } \\
\text { Generated } \\
(\mathrm{mW})\end{array}$ \\
\hline Test magnet & 340 & 3.5 & 760 & 17.5 & 1890 & 108.2 \\
\hline 2 & \multirow{3}{*}{\multicolumn{2}{|c|}{ Schedule for future studies }} & 110 & 0.34 & \multirow{3}{*}{\multicolumn{2}{|c|}{ Schedule for future studies }} \\
\hline 6 & & & 500 & 7.6 & & \\
\hline 10 & & & 2000 & 121.2 & & \\
\hline
\end{tabular}

\section{Table 1}

The quantity of energy harnessed from this work at this stage is still not sufficient to make a profitable commercial "real-world" system. We can produce better results by either making the elevator move faster or by increasing the number of magnets. Further issues that need to be considered include the prevention of the magnetic field affecting the passengers inside the elevator and reducing the cost of the materials. Although this prototype successfully proves the concept of harnessing energy from the vertical motion of an elevator, for it to be a commercially profitable product, it needs more work. We hope that the result from this educational model proposed in this paper would serve as a milestone, and help future innovators to use this concept and technology to produce a profitable "real-world" elevator to generate energy. The skills learned from this project were invaluable, as research, design, trial and error, as well as technical writing are all important experiences within engineering and physics. 


\section{Acknowledgement}

The authors would like to express their sincere thanks to Dr. Donald Goldthwaite and the First year Engineering Learning and Innovation Center and Lab Assistants for their support during this project and making the prototype in the lab.

\section{References}

1. History of Energy Use in the United States, https://geology.com/articles/history-of-energy-use/

2. Clean Revolution, Robert F. Service, Science, Vol. 350, Issue 6264, 2015

3. Electricity without Carbon, Quirin Schiermeier, Jeff Tollefson, Tony Scully, Alexandra Witze \& Oliver Morton, Nature, Vol 454, 816-823 (2008)

4. Renewable Energy Generation from Frequent Human Activity, Matthew Bonanni, Peter Groen, Brian Liang, Owen Porth, Spencer Pozder, and Bala Maheswaran, ASEE-NE 2016 Conference Proceeding.

5. Harnessing Energy from Everyday Movement: A Proposition for the Expansion of Piezoelectrics, Conor Byrne, Tim Cook, Emily Malterre, Vidhan Bhaiya, and Bala Maheswaran, ASEE-NE 2017 Conference Proceeding.

6. The Deskcycle: A Proposal for the Application of DC Generators towards Efficient, College Student Powered Energy Generation, Jack Carvalho, Edgar Castro, Erin Raftery, Jeffrey Yao, and Bala Maheswaran, ASEE-NE 2017 Conference Proceeding.

7. Renewable Energy Generation from Footsteps using Piezoelectronics, Matthew Hoffman, Molly Sharpe, Zachary Stern, Daniel Trapp, and Bala Maheswaran, ASEE-NE 2017 Conference Proceeding.

8. The Science of Teaching Science, M. Mitchell Waldrop, Nature, Vol 523, 272-274 (2015)

9. How much Physics the First Year Engineering Students really need: A Study and Survey, Bala Maheswaran, ASEE Conference Proceeding, AC 2012-3390.

10. Impact of a Design Project on Engineering Physics: Does motor design project motivate students? Bala Maheswaran, ASEE Conference Proceeding, AC 2013.

11. A New Teaching Approach for Ancient Engineering Physics: Master Physics via Mastering Physics! A Study and Survey, Bala Maheswaran, ASEE Conference Proceeding, AC 2014.

12. Physical and Virtual Laboratories in Science and Engineering Education, Ton de Jong, Marcia C. Linn, and Zacharias C. Zacharia, Science, Vol. 340, Issue 6130, 2013

13. Developing Interactive Teaching Strategies for Electrical Engineering Faculty, Margret Hjalmarson, Jill K Nelson, Lisa G. Huettel, Wayne T. Padgett, Kathleen E. Wage, and John R. Buck, ASEE Conference Proceedings AC 2013

14. University Physics with Modern Physics, by Young and Freedman, $14^{\text {th }}$ Edition, Pearson.

15. Faradays's Law - HyperPhysics Cocepts: http://hyperphysics.phyastr.gsu.edu/hbase/electric/farlaw.html

16. Elevator and Escalator Fun Fact: Copyright @ 2003-2018 National Elevator Industry, Inc., Centreville, VA

17. Regenerating the Energy from Building Lift, Achal Shahare and Badal E.Ganvir, International Journal for Research in Emerging Science and Technology, Volume-2, Issue-4, 2015

18. SIK Experiment Guide for Arduino. https://learn.sparkfun.com/tutorials/sik-experiment-guidefor-arduino---v333

19. Electrical Circuits Online: Towards a Completely Online Electrical Engineering Curriculum, Yacob Astatke, Craig J. Scott, and Jumoke 'Kemi Ladeji-Osias, ASEE Conference Proceedings AC 2011-1007 